\title{
La demande d'aide spécialisée des enseignants ordinaires du premier degré auprès des RASED
}

Feuilladieu Sylviane sylviane.feuilladieu@univ-amu.fr

Sociologie, Aix-Marseille Université, EA 4671 ADEF

Tambone Jeannette jane.tambone@wanadoo.fr

Sciences de l'éducation, Aix-Marseille Université, EA 4671 ADEF

\section{Résumé}

Une enquête sur les pratiques déclarées de demandes d'aides spécialisées, que les enseignants ordinaires adressent aux membres des RASED concernant leurs élèves en difficulté, met au jour le travail conjoint mené à cette occasion. Apparaît ainsi un travail invisible mais néanmoins essentiel de régulation des demandes d'interventions spécifiques, mais aussi du regard que peut porter un enseignant sur les difficultés d'apprendre de ses élèves, et sur sa propre difficulté à enseigner. Si le RASED ne modifie pas la forme scolaire du système éducatif et représente en ce sens la bonne conscience d'un système inégalitaire, le travail conjoint qu'il engendre dans les écoles ouvre toutefois un espace possible d'émancipation et de réduction des inégalités scolaires.

Mots clés : Dispositif d'aide spécialisée - Élèves en difficulté - Enseignants - RASED Régulation - Travail collectif. 
De multiples dispositifs organisent l'aide aux élèves en difficulté, tant dans le premier que le second degré. Deux logiques sous-tendent les différentes formes d'aides (Suchaut, 2009): une logique basée sur les moyens supplémentaires alloués à une population particulière (éducation prioritaire...), et une logique visant les pratiques et les relations pédagogiques (activités pédagogiques complémentaires...). Les réseaux d'aides spécialisées aux élèves en difficulté (RASED) qui interviennent dans le premier degré, rassemblent ces deux logiques, en mobilisant à la fois des moyens et des pratiques spécifiques. Trois types d'aides sont proposées sur le temps scolaire : à dominante pédagogique (maîtres $\mathrm{E}$ ), à dominante rééducative (maîtres $\mathrm{G}$ ), et psychologique (psychologues). L'enquête présentée ici s'appuie sur les pratiques déclarées de demandes d'aides spécialisées que les enseignants ordinaires adressent aux membres des RASED, concernant leurs élèves en difficulté.

\section{Le contexte institutionnel des RASED}

Les RASED sont créés en 1990 afin de proposer une aide spécialisée aux élèves en difficulté, lorsque la réponse du maître de la classe dans le cadre d'une pédagogie différenciée n'a pas été efficiente $(M E N, 1990)^{1}$. La circulaire précise que l'existence des aides spécialisées ne doit pas provoquer une multiplication excessive de demandes. L'intervention du RASED s'effectue après une mise en commun des résultats des différentes approches effectuées, et impliquent une collaboration étroite ainsi qu'une information réciproque entre les enseignants, les intervenants spécialisés, et autres professionnels. Afin de réguler les demandes d'aide, les membres des RASED ont développé sur les terrains des documents écrits, proposant aux enseignants ordinaires de préciser les difficultés de l'élève et ce qui a déjà été mis en place pour lui. Ce n'est qu'en 2002, dans la nouvelle circulaire qui définit les dispositifs d'adaptation et d'intégration scolaires, que sont évoquées et préconisées les procédures formelles de demande d'aide. «L'intérêt de ce travail réside dans l'élaboration collective conduisant à expliciter des critères communs, à éclaircir les registres dans lesquels les difficultés se manifestent, ainsi que les éléments positifs sur lesquels s'appuyer. C'est pourquoi cette formalisation des procédures ne se réduit pas à la rédaction d'une fiche, et ne se substitue pas aux échanges indispensables entre les parties prenantes permettant la confrontation et la synthèse d'approches plurielles» (MEN, 2002). L'efficacité de la mobilisation des ressources spécifiques dans l'école repose sur la collaboration des différents partenaires et la cohérence de leurs interventions. La circulaire de 2009 sur les fonctions des personnels spécialisés des RASED dans le traitement de la difficulté scolaire à l'école primaire, confirme leurs compétences spécifiques au sein des équipes pédagogiques,

\footnotetext{
${ }^{1}$ L'objet n'est pas ici de proposer un historique des RASED ni de l'enseignement spécialisé, mais de situer le travail réalisé dans I'évolution du contexte institutionnel.
} 
Iorsque l'aide personnalisée ou les stages de remise à niveau se révèlent insuffisants ou inadaptés (MEN, 2009). Pourtant, dans le même temps, les RASED connaissent une période de suppressions importantes de postes. La fédération nationale des rééducateurs de l'éducation nationale (maîtres $G$ ), sur la base des chiffres ministériels, estime une diminution d'environ un tiers des postes entre 2008 et 2012 (5000 sur 14889, principalement des maîtres E et G ; FNAREN, 2013). Le contexte a aujourd'hui évolué. Dans le cadre de la loi de refondation de l'école de 2013, les premières décisions relatives à l'évolution des métiers de l'éducation nationale repositionnent la pertinence du travail des RASED (MEN, 2014a). Face à la persistance d'une proportion non négligeable d'élèves ne maîtrisant pas les compétences de base du socle commun de connaissances et de compétences, la politique éducative affichée est de « donner à chacun les moyens de mieux apprendre » (MEN, 2013). 12\% des élèves ne réussissent pas les évaluations nationales en français à la fin du premier degré (34\% chez les élèves en retard), $9 \%$ en mathématiques (29\% chez les élèves en retard; MEN, 2014c). Les directives institutionnelles oscillent entre deux visions des RASED. D'une part, des prises en charge déconnectées des classes, coupant davantage les élèves en difficulté des apprentissages qui s'y déroulent, et dont l'efficacité sur les trajectoires scolaires n'est pas démontrée (Gossot, 1997 ; Ferrier, 1998). D'autre part, une place et un rôle spécifiques dans l'aide aux élèves, et dans l'appui et l'accompagnement des équipes, dont l'intérêt est réaffirmé (MEN, 2014b). Une vision contrastée en lien avec les tensions qui traversent l'espace symbolique occupé par les RASED, allégorie d'un système éducatif coincé entre sélectivité et progression de tous (espace représentant de manière métaphorique cette contradiction). De cette place périphérique les RASED jouent donc, paradoxalement, un rôle central : permettre au système de fonctionner et d'assurer sa mission démocratique, enseigner à tous les élèves. Dans ce contexte, il nous semble important de revenir sur le travail collaboratif entre enseignants ordinaires et membres des RASED, posé comme condition nécessaire des interventions spécifiques et de leur efficacité, auprès des élèves dont les difficultés d'apprentissage ont résisté aux aides apportées par les enseignants des classes. L'ultime recours en quelque sorte des équipes des écoles face à ce type de difficultés, avant que le système, impuissant à les résoudre, ne délègue ce problème à des professionnels extérieurs à l'éducation nationale. L'enquête proposée dans cet article a été menée en 2000, au moment où la demande d'aide spécialisée n'était pas encore institutionnalisée dans les textes, mais formalisée dans les pratiques par les professionnels. Ces données apportent ainsi un éclairage intéressant, sur la création des outils et l'organisation concrète des conditions de travail permettant la régulation et l'efficience des interventions spécifiques.

\section{Inventer conjointement pour résoudre collectivement}


Les interventions du RASED sont externes à la classe. La remédiation des obstacles rencontrés dans le dispositif central d'apprentissage (la classe), est en ce sens externalisée, et confiée à un dispositif auxiliaire (le RASED). Elle ne peut donc être pensée indépendamment de la classe, au risque d'inefficacité et d'inefficience (Tambone \& Mercier, 2003). En effet pour être efficace (résultats obtenus conformes aux objectifs attendus), un dispositif doit être efficient (utilisation pertinente des ressources en vue des objectifs attendus). Dispositif est entendu ici comme un « ensemble de moyens organisés, définis et stables, qui sont le cadre d'actions réitérables, conduites pour répondre à un problème récurrent » (Chartier, 1999). En l'occurrence, le problème récurrent est la double difficulté d'enseigner et d'apprendre en classe ordinaire. Le réseau constitue le cadre des actions réitérables de remédiation, et la demande d'aide l'acte déclencheur de définition et d'organisation des moyens pouvant répondre à ce problème. Le travail conjoint qui s'instaure ainsi autour de cette demande entre les enseignants ordinaires et les membres des RASED, constitue une dynamique collective « qui crée des dépendances d'action réciproques orientées par les apprentissages des élèves. Il ne s'agit pas seulement d'accords communs, mais d'une action partagée qui solidarise les acteurs dans leurs gestes professionnels » (Mérini \& Ponté, 2009). Loin d'être une simple sollicitation-réponse, la demande d'aide relève de, et impacte, différentes dimensions du métier et différents temps scolaires : le temps calendaire (emplois du temps, moments où I'enseignant ordinaire s'adresse au RASED, où le RASED reçoit les demandes), I'organisation de l'institution (rythme de la classe et des programmes d'un côté, rythme d'apprentissage des élèves de l'autre), les échanges collaboratifs (travailler ensemble), la construction de l'identité professionnelle (définition et répartition des rôles entre adultes, et vis-à-vis des élèves) (Dorison, 2006 ; Ponté, Thomazet \& Mérini, 2012). De plus, si les prescriptions multiplient le nombre de dispositifs d'aide aux élèves en difficulté, elles n'établissent pas de manière précise les objectifs à atteindre, les moyens pour les atteindre, et I'organisation concrète du travail collectif. Ainsi, une part des pratiques repose sur l'inventivité des professionnels, «sans qu'ils puissent pour autant mettre en œuvre des règles communes et reconnues de tous, issues de l'histoire du métier enseignant » (Félix, Saujat \& Combes, 2012). La demande d'aide est donc une pratique privilégiée pour observer : comment les enseignants ordinaires décrivent les difficultés de leurs élèves et leurs limites d'enseignement (résultats connexes en cours de publication); la production et la mobilisation de règles effectives de travail permettant l'échange social et l'action collective au sein de l'institution, confrontée à un problème pratique (Reynaud, 1997). Ces règles effectives de travail sont un compromis entre les règles imposées par les autorités (textes officiels de l'éducation nationale) et celles qu'admettent les usagers (routines négociées entre les acteurs concernés). Elles constituent un moyen de résoudre ce problème pratique, à partir du cadre de travail 
posé, et une solution à ce problème, à travers leur mise en œuvre concrète et productrice des échanges nécessaires à sa résolution. L'aide aux élèves en difficulté exige donc un véritable projet collectif, sa délégation aux seuls membres des RASED n'étant pas opérante. L'élaboration du travail conjoint nécessite la traduction réciproque entre enseignants ordinaires et membres des RASED des difficultés rencontrées, de manière à les définir comme problème commun, et la négociation des modalités de collaboration permettant d'engager et de mobiliser tout le monde (Callon, 1985). Comment s'organisent les demandes d'aide spécialisée dans les écoles ?

\section{Les pratiques déclarées des demandes d'aides}

Une enquête par entretiens semi directifs a été menée auprès de 60 enseignants du premier degré, 30 enseignants ordinaires (cycles 1, 2, 3) et 30 membres des RASED (maîtres $E$, maîtres $G$, psychologues). Tous ces professionnels ont été amenés à décrire la manière dont ils signalent les élèves en difficulté, ou la manière dont ils leur sont signalés. Bien que l'enquête porte sur les demandes d'aide, le terme utilisé dans les entretiens est celui de «signalement», dans un souci de clarté et d'accessibilité langagières pour les professionnels interrogés (Ghiglione \& Matalon, 1985 ; ce terme étant très usité dans les pratiques bien que non institutionnalisé, comme l'attestent diverses publications ${ }^{2}$ ). Une analyse lexicométrique a été effectuée à l'aide du logiciel de statistique textuelle Alceste. Les résultats présentés portent sur les formes lexicales évoquant directement le signalement et le travail qui s'organise autour : «signaler» «signalement» (toutes formes conjuguées, singulier, pluriel), «fiche » «feuille » (singulier, pluriel), «difficulté » (singulier, pluriel), « regard »(singulier, pluriel). Une analyse thématique de contenu a ensuite été réalisée sur tous les énoncés contextuels de ces formes. Les unités sémantiques cohérentes qui se dégageaient de ces contextes discursifs ont été catégorisées selon les principes de classement de Bardin (les catégories doivent être exhaustives, exclusives, méthodiques, objectives, quantifiables, 1989). Le nombre d'énoncés ainsi répertoriés, a fait l'objet d'une comparaison entre enseignants ordinaires et enseignants spécialisés, sur la base du nombre d'occurrences des formes lexicales.

\footnotetext{
2 Bélanger, N. (2006). Les processus de signalement de l'enfance en difficulté à l'école de langue française en Ontario. $8^{\text {ème }}$ Biennale de l'éducation et de la formation. Paris : INRP, APRIEF. [www.inrp.fr/biennale/8biennale/contrib/longue/349.pdf] Del Notaro, L. (1997). Analyse des fiches de signalement des élèves en difficulté à l'école primaire du Canton Tessin. Genève : Faculté de psychologie et des sciences de l'éducation, Rapport de recherche.

Guimard, P. (2004). Effets de l'âge et du signalement sur les performances scolaires : étude exploratoire au cycle 2. Approche Neuropsychologique des Apprentissages chez l'Enfant, 143-152.

Pecaut, M. (1998). La représentation du pair face à un enfant signalé. Université de Bordeaux 2 : Mémoire pour l'obtention du Diplôme d'Etat de Psychologie Scolaire.

Robineau, C. (1996). Les jugements scolaires : le signalement des élèves en difficultés par les enseignants du premier degré. Université de Bordeaux 2 : Mémoire pour l'obtention du Diplôme d'Etat de Psychologie Scolaire.

Schlanser G. (2002). Le signalement des élèves en difficulté. La nouvelle revue de l'AIS, nº17.
} 


\section{Une régulation qui se construit dans le temps}

De manière générale, l'utilisation des termes « signaler » et « signalement » caractérise majoritairement le discours des membres des RASED. Celui-ci comporte 76\% des 1141 occurrences, soit 3 fois plus que le discours des enseignants ordinaires, quel que soit le thème abordé $\left(\mathrm{X}^{2}{ }_{(5)}=39.21, \mathrm{p}=.01\right.$, tableau 1$)$.

\begin{tabular}{|l|c|c|c|c|c|c|}
\hline Signaler - Signalement & \multicolumn{2}{|c|}{$\begin{array}{c}\text { Enseignants } \\
\text { Ordinaires }\end{array}$} & \multicolumn{2}{|c|}{$\begin{array}{c}\text { Membres } \\
\text { RASED }\end{array}$} & \multicolumn{2}{c|}{$\begin{array}{c}\text { Total nombre } \\
\text { occurrences }\end{array}$} \\
\hline 1- Destinataire & 52 & $36 \%$ & 94 & $74 \%$ & 146 & $100 \%$ \\
\hline 2- Modalités et déroulement & 47 & $14.5 \%$ & 278 & $85.5 \%$ & 325 & $100 \%$ \\
\hline 3- Motifs & 112 & $29.5 \%$ & 268 & $70.5 \%$ & 380 & $100 \%$ \\
\hline 4- Points de vue sur... & 11 & $15 \%$ & 62 & $85 \%$ & 73 & $100 \%$ \\
\hline 5- Travail collectif déclenché & 29 & $20 \%$ & 115 & $80 \%$ & 144 & $100 \%$ \\
\hline 6- Pas de signalement & 22 & $30 \%$ & 51 & $70 \%$ & 73 & $100 \%$ \\
\hline Total & $\mathbf{2 7 3}$ & $\mathbf{2 4} \%$ & $\mathbf{8 6 8}$ & $\mathbf{7 6} \%$ & $\mathbf{1 1 4 1}$ & $\mathbf{1 0 0} \%$ \\
\hline
\end{tabular}

Tableau 1 : Catégorisation des énoncés contextuels des formes « signaler » et « signalement », comparaison entre enseignants ordinaires et membres des RASED

Concernant les modalités concrètes de signalement, les professionnels évoquent surtout une pratique formelle, notamment à travers l'importance de sa dimension écrite (46\% des occurrences, soit 150 sur 325 ; c'est 3.5 fois plus que la description de pratiques informelles ou orales qui rassemble 43 occurrences). La formalisation de cette pratique passe par le remplissage d'une fiche dédiée, comportant diverses rubriques regroupant des informations sur l'élève signalé (âge, parcours scolaire...), le type et le niveau de difficultés (mathématiques, français, consignes, comportement...), les causes probables et/ou supposées des difficultés (problèmes de compréhension, problèmes familiaux...), les aides mises en place par l'enseignant (soutien scolaire, étayages et outils proposés en classe...), les rencontres avec les parents... Cette fiche est à l'initiative des membres des RASED. $90 \%$ des occurrences des termes «fiche» et «feuille», supports du signalement, proviennent de leur discours (223 occurrences vs 24 chez les enseignants ordinaires, sur un total de 247). Les membres des RASED souhaitent recueillir les informations nécessaires à l'analyse des difficultés des élèves signalés et à l'élaboration du projet d'aide. Ils incitent ainsi les enseignants ordinaires à prendre du recul, à travers le passage à l'écrit, vis-à-vis des premières impressions formulées à la rentrée, comme I'explique cet enseignant spécialisé chargé des aides rééducatives : "Il est vraiment effectif, le signalement, quand il y a la fiche de signalement, l'écrit, quand on est passé 
par cet acte où il y a eu une réflexion, où on a écrit des choses. Donc là il y a une trace de ce qui a été dit, c'est important pour aussi après justement la fin de la rééducation, de voir un peu à partir de ce signalement initial ce qui s'est passé, que si la personne justement devait à nouveau remplir la fiche, est-ce qu'elle dirait la même chose ? Donc le signalement pour moi c'est là, c'est ce moment où la fiche est rédigée».

Les membres des RASED font de cette fiche un outil de régulation des premières demandes d'aide, et organisent à cette fin le signalement en plusieurs temps, alternant rencontres avec les enseignants et pauses de réflexion. Les passages suivants, extraits d'entretiens avec des enseignants spécialisés chargés des aides rééducatives, illustrent ce type de déroulement : «Moi ce signalement, enfin ce signe qu'on nous fait comme ça lorsqu'on traverse un couloir : "à propos, il faudrait que je te parle de...", bon c'est un appel quelque part. Après, le signalement vient après. Moi je pense que quand il y a, justement on en rediscute, alors ça peut-être oral, on essaie d'approfondir un peu. »; «On va tourner dans toutes les écoles, ce qui nous fait entre 90 et 100 classes à voir et quand on revient un mois plus tard, parce qu'il faut presque un mois pour faire ça, déjà les signalements changent. (...) On peut entendre parler d'enfants dont on nous a pas parlé du tout, qui ont été carrément occultés. Et il arrive même que l'instit dès qu'elle nous voit : "ah! j'ai oublié de te parler de, alors que c'est lui qui m'embête le plus". (...) Et il peut y avoir inversement, il y a aussi tout simplement des enfants, ils disent : "ça va mieux"». Ces propos témoignent de l'importance des modalités concrètes du signalement dans les écoles, dans le réajustement des difficultés signalées et de la définition du travail de chacun. Un enseignant spécialisé chargé des aides pédagogiques évoque l'intérêt de telles explicitations entre les différents professionnels : «Au niveau du signalement y'a rien d'écrit. D'ailleurs ça m'interpelle un peu parce que je pense que je vais évoluer vers quelque chose de plus écrit peut-être, de plus institutionnalisé. Pour essayer de clarifier les choses notamment par rapport aux instits pour que leur demande soit plus précise. Parce que bon il y a toujours un petit effet, souvent elles évaluent les gamins sur leur comportement en classe, et sur leur comportement non pas scolaire mais un comportement d'élève quoi.». Associée dans les textes au travail mené par les RASED, I'organisation de ces modalités leur incombe et entraîne pour eux la nécessité de créer les conditions de leur intervention (63 occurrences relatent le déroulement local et temporel du signalement dans leur discours vs 1 dans le discours des enseignants ordinaires, parmi les 325 de la catégorie « modalités et déroulement » du tableau 1).

\section{Des regards pour un nouveau regard}

Le réajustement des signalements, à travers les modalités formelles et différées mises en place par les RASED, engendre des échanges de points de vue entre les professionnels, permettant de passer d'un regard singulier à un regard pluriel sur les élèves en difficulté. 
Les points de vue échangés évoluent vers une analyse plus complète et une compréhension plus fine des problèmes rencontrés, comme en témoignent cet enseignant spécialisé chargé des aides pédagogiques, et ce psychologue scolaire : « Je pense que plus on a de regards sur l'enfant, plus on a des points de vue différents, et plus on est obligé de discuter et par conséquent de prendre en compte l'intérêt de l'enfant. Voilà. Voilà le rôle du réseau. »; «Il y a tout ce travail aussi à... à faire auprès des enseignants, c'est-à-dire de changer le regard de l'enfant, sur l'enfant en difficulté. ». Ce changement de regard agit à deux niveaux. Au niveau de l'élève il permet d'intégrer la dimension personnelle à la dimension scolaire, en resituant le fait que I'élève est un enfant qui va à l'école. Ponté, Thomazet \& Mérini (2012) considèrent que «cette stratégie du déplacement du regard du maître ordinaire en l'invitant à prendre en compte I'histoire d'un élève semble être une constante du métier, susceptible d'être qualifiée de geste professionnel du maître $E$ ». Au niveau de l'enseignant, il permet une nouvelle mise en perspective des obstacles rencontrés en classe, en élargissant les capacités d'expertise et les possibilités pratiques. L'enseignant peut même prendre conscience que certains de ces obstacles s'enracinent dans les situations pédagogiques proposées. L'activité du RASED aide à apprendre, à comprendre, à enseigner, à rompre I'isolement des enseignants. Le RASED dynamise le travail collectif de l'équipe de l'école au service de tous. Ainsi l'expriment cet enseignant de cycle 3 et cet enseignant spécialisé chargé des aides pédagogiques : «Nous on est seul dans notre classe, bon c'est sûr qu'il y a des choses qu'on voit et d'autres qu'on laisse passer ; parce que bon, bien... Et c'est pour ça que le regard d'une autre personne c'est intéressant, sur l'enfant. »; « Le fait d'ouvrir sa classe à un regard extérieur, le fait de s'entendre dire que bon ailleurs ça se passe autrement, quelque part ça l'a sécurisée quoi. Voilà, je pense que là le réseau aussi, le signalement a permis un certain travail avec cette maîtresse-là. ». L'action des RASED sur la difficulté s'opère au cœur du travail conjoint, et pas seulement lors des séances de remédiations. En ce cas, la demande d'aide fait l'objet d'un travail partagé au sens où l'activité professionnelle est « effectuée ensemble à plusieurs », notion qui « recouvre l'ensemble des pratiques enseignantes autres que les pratiques d'enseignement: les pratiques de collaboration et de coopération au sein de différents dispositifs mais aussi les pratiques collectives dans le cadre formalisé de conseils ou peu formalisés de la vie de l'établissement » (Marcel \& Garcia, 2009).

\section{Le rapport des enseignants à la difficulté}

$19 \%$ des énoncés contextuels des termes « difficulté(s) 》, évoquent le rapport personnel de l'enseignant à sa propre difficulté d'enseigner (135 occurrences sur 709 ; thème majoritairement présent dans le discours des membres des RASED : 110 occurrences vs 25 dans celui des enseignants ordinaires). Même si certains enseignants hésitent à 
signaler un élève au RASED par crainte d'être jugés, beaucoup d'entre eux saisissent ce dispositif pour exprimer leurs limites, leur sentiment d'incapacité, d'échec et d'épuisement, comme en témoignent ces professeurs des cycles 2 et 3 : «bon ben maintenant j'ai testé l'enfant, je connais ses difficultés mais je n'ai pas le temps de m'en occuper. Il faut qu'il soit vu à l'extérieur, moi je suis saturée et je ne peux plus »; «moi je ne peux pas travailler ni avec sa maman, ni avec son papa et personne d'autre non plus, c'est, ça nous dépasse complètement. Il y a des limites finalement, ça aussi, il y a des limites quand on veut aider un enfant en difficulté, je trouve qu'il y a des limites parce que je prends son cas à lui, moi je le vis comme une situation d'échec parce que je ne sais plus quoi faire ». Un psychologue scolaire parle de SOS lancés : « et puis bon parfois les difficultés très très lourdes, majeures, importantes apparaissent d'emblée. Il n'y a pas d'autres, comment dirai-je ? Là c'est un cri, c'est un signal, c'est une alarme, c'est : SOS, c'est : "au secours"! Il y a plusieurs types de signalements et puis on a opté pour entendre tout ce qu'ils [les enseignants] veulent dire sur chaque enfant ».

Cette dimension d'aide à l'enseignant, dans le cadre du travail des RASED, montre I'ambigüité de ce type de dispositifs, entre outil de domination sociale et espace d'émancipation et de réduction des inégalités scolaires. D'un côté parce qu'ils sont externes à la classe, ils ne permettent pas une remise en cause suffisante d'un système éducatif unique qui s'adresse à tous, mais reste pensé pour les meilleurs. Ils maintiennent cette dualité élitiste entre les élèves qui réussissent et les autres, « adaptation et indifférence aux différences, en ce sens, sont deux facettes de la même idéologie sélective»(Bonnéry, 2007). De l'autre, parce qu'ils ouvrent un espace de parole et d'action collective où peuvent se réguler les insuffisances d'une gestion bureaucratique et « des formes traditionnelles d'action, qui s'adressent à des catégories collectives peu différenciées et mettent en œuvre des réponses stéréotypées » peu adaptées (Demailly, 2008), ils autorisent la reconnaissance et la restauration des dignités bafouées par l'échec scolaire (Bourdieu, 1993). Ainsi, le RASED est à la fois la bonne conscience du système, et la soupape indispensable à sa survie.

\section{L'espace paradoxal d'un système démocratique inégalitaire}

Les données montrent que l'activité du RASED exerce une double fonction d'aide, auprès des élèves et des enseignants. Elle impacte sur la double difficulté d'apprendre et d'enseigner. Une étude sur les mesures d'aide montre l'importance d'un tel réseau de relations dans les établissements scolaires entre les enseignants des classes et des collègues-ressource : «les enseignants incluant dans leur classe des élèves en difficulté sont plus à risque d'épuisement professionnel... Un des facteurs de protection efficace contre le burn-out est le soutien social, c'est-à-dire le réseau d'aide qu'une personne peut solliciter lorsqu'elle est confrontée à des situations professionnelles 
problématiques »(Doudin, 2009). Un enseignant ordinaire de cycle 1, interrogé dans notre enquête, l'exprime ainsi : «avec ces enfants-là il faut pas du bricolage. J'ai l'impression qu'il faut vraiment quelque chose de très structuré et des connaissances bien, bien posées. Voilà. Alors pour moi c'est un handicap. Moi aussi je suis en difficulté totale par rapport à ça. Je me signale ».

Les enseignants doivent concilier l'inconciliable : I'intégration de tous les élèves dans un tronc commun qui vise l'efficacité des résultats, avec la prise en compte différenciée des besoins particuliers des élèves en difficulté au cœur de programmes et d'évaluations standardisés (Maulini \& Mugnier, 2012). Les études PISA menées dans les pays de I'OCDE montrent que s'ajustent l'équité et l'efficacité des systèmes éducatifs. Les Ecoles de masse sont souvent les Ecoles d'excellence : celles qui comptent beaucoup de bons élèves comptent moins d'élèves en échec et moins d'inégalités sociales. L'élévation du niveau général de la masse participe de l'élévation générale du niveau (Baudelot \& Establet, 2009). Dans l'attente d'un système plus équitable et plus efficace, le travail conjoint entre enseignants ordinaires et membres des RASED ouvre au sein de l'espace scolaire un lieu possible d'efficience pédagogique et de reconstruction des identités abimées (Payet, 1994).

\section{Bibliographie}

Bardin, L. (1989). L'analyse de contenu. Paris : PUF.

Baudelot, C., Establet, R. (2009). L'élitisme républicain. Paris : Seuil.

Bonnéry, S. (2007). Comprendre l'échec scolaire. Elèves en difficultés et dispositifs pédagogiques. Paris : La Dispute.

Bourdieu, P. (1993). La misère du monde. Paris : Seuil.

Callon, M. (1986). Eléments pour une sociologie de la traduction. L'année sociologique, 36, 57-63.

Chartier, A-M., (1999). Un dispositif sans auteur : cahier et classeurs à l'école primaire. Hermès, 25, 207-218.

Demailly, L. (2008). Politiques de la relation. Approche sociologique des métiers et activités professionnelles relationnelles. Paris : Presses universitaires du Septentrion.

Dorison C., (2006). La place de la pédagogie dans les relations entre enseignants spécialisés et enseignants des classes ordinaires : du tabou à la collaboration. Les sciences de l'éducation pour l'ère nouvelle, 38, 2, 63-78.

Doudin, P-A., Lafortune, L., Pons, F., Moreau, J. (2009). Le paradoxe de certaines mesures d'aide à I'intégration scolaire : quand l'aide mène à l'exclusion. Revue des sciences de l'éducation, 35, 1, 41-53.

Félix, C., Saujat, F., Combes, C. (2012). Des élèves en difficulté aux dispositifs d'aide : une nouvelle organisation du travail enseignant ? Recherches en éducation, 4, 19-30.

Ferrier, J. (1998). Améliorer l'efficacité de l'école primaire. Rapport au Ministère de l'éducation nationale. Paris : La documentation française.

FNAREN (2013). RASED: état des postes depuis 2008. Document du bureau national. [http://dcalin.fr/fichiers/rased_effectifs.pdf] 
Ghiglione, R., Matalon, B. (1985). Les enquêtes sociologiques. Paris : A. Colin.

Gossot, B. (1997). Les réseaux d'aides spécialisées aux élèves en difficulté : examen de quelques situations départementales. Rapport du groupe de l'enseignement primaire de I'Inspection Générale de I'Education Nationale. Paris : MEN.

Marcel, J-F., Garcia, A. (2009). Contribution à une théorisation des interrelations entre les pratiques enseignantes de travail partagé et les pratiques d'enseignement. Les sciences de l'éducation pour l'ère nouvelle, 42, 2, 27-42.

Maulini, O., Mugnier, C. (2012). Entre éthique de l'intégration et pratiques de la différenciation : (re)penser I'organisation du travail scolaire ? Recherches en éducation, 4, 9-18.

MEN-Ministère de l'éducation nationale (2014a). La refondation de l'école : premières décisions sur l'évolution des métiers de l'éducation nationale. [http://www.education.gouv.fr/cid75724/ premieres-decisions-sur-I-evolution-des-metiers-de-I-education-nationale.html]

MEN (2014b). La refondation de l'école : fiches sur l'évolution des missions et des métiers des personnels de l'éducation nationale. [http://www.education.gouv.fr/ cid77283/toutes-les-fichessur-I-evolution-des-missions-et-des-metiers-des-personnels-de-l-education.html]

MEN (2014c). Repères et références statistiques. [http://www.education.gouv.fr/cid57096/repereset-references-statistiques.html]

MEN (2013). Année scolaire 2013-2014: la refondation de l'École fait sa rentrée. [http://www.education.gouv.fr/cid73417/annee-scolaire-2013-2014-la-refondation-de-I-ecolefait-sa-rentree.html]

MEN (2009). Fonctions des personnels spécialisés des RASED dans le traitement de la difficulté scolaire à l'école primaire. Circulaire n²009-088 du 17/7/2009. Paris : Bulletin Officiel n³1 du $27 / 8 / 2009$.

MEN (2002). Les dispositifs de l'adaptation et de l'intégration scolaires dans le premier degré. Circulaire n॰2002-113 du 30/4/2002. Paris : Bulletin Officiel n 19 du 09/05/2002.

MEN (1990). Mise en place et organisation des réseaux d'aides spécialisées aux élèves en difficulté. Circulaire n॰90-082 du 09/4/1990. Paris : Bulletin Officiel n 16 du 19/4/1990.

Mérini, C., Ponté, P. (2009). Le travail conjoint à l'école : exploration des modalités d'action. Les sciences de l'éducation pour l'ère nouvelle, 42, 2, 43-65.

Payet, J-P. (1994). L'école à l'épreuve de la réparation sociale : la relation professionnels-public dans les établissements scolaires de banlieue. Revue française de pédagogie, 109, 7-17.

Ponté, P., Thomazet, S., Mérini, C. (2012). Au seuil de la classe, le maître E régule différentes temporalités. Recherches en éducation, 4, 31-41.

Reynaud, J-D. (1997). Les règles du jeu. Paris : A. Colin.

Suchaut, B. (2009). L'aide aux élèves: diversité des formes et des effets des dispositifs. Communication aux $2^{2 \mathrm{e} m e}$ rencontres nationales sur l'accompagnement. France : St Denis.

Tambone, J., Mercier, A. (2003). L'articulation entre classe et groupe d'adaptation de l'aide à dominante pédagogique, en France, pose questions sur la notion de système didactique. In Chatelanat, G. \& Pelgrims, G. (Éds.). Éducation et enseignement spécialisés : ruptures et intégrations. Bruxelles : De Boeck. 